\title{
Three-Dimensional Modeling of Wave Propagation over Different Types of Terrains and Environments Using the Parabolic Equation Solved by Higher Order Approximation of the Finite Difference Method
}

\author{
Mohammad Ahmad ${ }^{1}$, Dayalan Kasilingam ${ }^{2}$ \\ ${ }^{1,2}$ Department of Electrical and Computer Engineering, University of Massachusetts, Dartmouth, USA
}

\begin{tabular}{l}
\hline \hline Article Info \\
\hline Article history: \\
Received Nov 11, 2020 \\
Revised May 15, 2021 \\
Accepted May 20, 2021 \\
\hline
\end{tabular}

\section{Keywords:}

Wave propagation

Three dimensional

Parabolic equation

Finite difference method

\begin{abstract}
Designing communications and radar systems depends on accurate modeling of ground waves in three-dimensional environment. Propagation of ground waves in the VHF and UHF bands affected by the characteristics of the terrain and the troposphere. Although some three-dimensional modeling of ground waves was found in the literature based on solving the parabolic equation, they were limited to a specific terrain and/or environment. Also, a lot of important factors such as the refractive index of the troposphere were ignored. In this paper, a computational model was developed for predicting the electromagnetic wave propagation over different types of terrains and environments under three-dimensional conditions. The model is based on solving the parabolic equation using higher order approximation of the finite difference method. The model allows specifications of an antenna and the electrical characteristics of the ground. Moreover, the model treats flat and non-flat terrains, mixed path with different electrical characteristics, and forest environment. Furthermore, the model enables calculations to be performed under standard and non-standard refractive conditions of the troposphere that varies in height, width, and range. The results were compared with twodimensional parabolic equation solved by Fourier split-step and showed excellent agreement.
\end{abstract}

Copyright $(2021$ Institute of Advanced Engineering and Science. All rights reserved.

\section{Corresponding Author:}

Mohammad Ahmad,

Department of Electrical and Computer Engineering,

University of Massachusetts,

Dartmouth, USA.

Email: mahmad1@umassd.edu

\section{INTRODUCTION}

Modeling of ground waves in three-dimensional (3D) environment is a major planning and design problem in communications and radar systems. This is because it gives a complete picture of the field variation in all directions at any desired point. Ground waves depend on characteristics of the terrain, environment, and the atmosphere lower layers.

One important and effective method used to model wave propagation is based on the parabolic equation (PE) [1]-[2]. This is because it provides complete wave solution for the field in the existence of terrain and environment that dependent on range, altitude, and width. Also, the solution of PE exhibit excellent robustness and accuracy for complicated problems involving 3D varying in troposphere refractive index.

A few 3-D implementations based on PE are found in the literature [3]-[11]. These methods have been applied only over restricted specification of an environment. Other environments and/or parameters that affect the propagation such as the refractive index for the troposphere, mixed surfaces environment, and forest environment have been ignored and/or not considered or tested. 
In this paper, the 3D PE is solved via higher order approximation of finite difference method (FDM) to model wave propagation over different types of terrains and environments under 3D conditions.

\section{RESEARCH METHOD}

\subsection{The parabolic equation}

Figure 1 shows the 3D environment to be considered. The 3D PE is defined by [10]:

$$
\frac{\partial^{2} f}{\partial z^{2}}+\frac{\partial^{2} f}{\partial y^{2}}+2 j k_{0} \frac{\partial f}{\partial x}+k_{0}^{2}\left(n^{2}-1\right) f=0
$$

where $x, y$, and $z$ are the range, altitude and width coordinates, $k_{0}=\omega / c$ is the free space wave number with $\omega=2 \pi f_{r}$ is the angular frequency with $f_{r}$ is the frequency and $c=3 \times 10^{8} \mathrm{~m} / \mathrm{sec}$ is the speed of light, and $n$ is the refractive index. Incorporating the effect of the troposphere and forest in (1) is done through $n$. For the troposphere, it is done via the relation between modified refractive index $M$ and the refractive index $n$ as $M(x, y, z)=\left(n(x, y, z)-1+\frac{y}{a}\right) 10^{6}$ where $a$ is the earth radius taken to be $6371 \mathrm{Km}$ and $y$ is the altitude in $\mathrm{Km}$. For standard refractivity $\partial M(x, y, z) / \partial y=0.118 \mathrm{M}$-units per meter. Incorporating the forest will be discussed in section 2.5.3. Equation (1) is limited to propagation angles less than $15^{\circ}$ above or below the local horizontal.

The transverse boundary condition (BC) over Earth's surface is a mixed BC expressed as

$$
\left(\alpha_{1} \frac{\partial}{\partial y}+\alpha_{2}\right) f(x, y, z)=0
$$

where $\alpha_{1}$ and $\alpha_{2}$ are constants. For lossy Earth's surface, $\alpha_{1}=1, \alpha_{2}=j k_{0} \sqrt{\varepsilon-1}$ and $\alpha_{1}=1, \alpha_{2}=$ $j k_{0} \sqrt{\varepsilon-1} / \varepsilon$ for horizontal polarization (HP) and vertical polarization (VP), respectively where $\varepsilon=\varepsilon_{r g}+$ $j 60 \sigma_{g} \lambda$ with $\varepsilon_{r g}$ and $\sigma_{g}$ are relative permittivity and conductivity of the surface ground at range $x$ and width $z$, and $\lambda=c / f_{r}$ is the wave length. For the perfectly electrical conductor (PEC) surface, $\alpha_{1}=0$ corresponding to the HP and $\alpha_{2}=0$ corresponding to the VP.

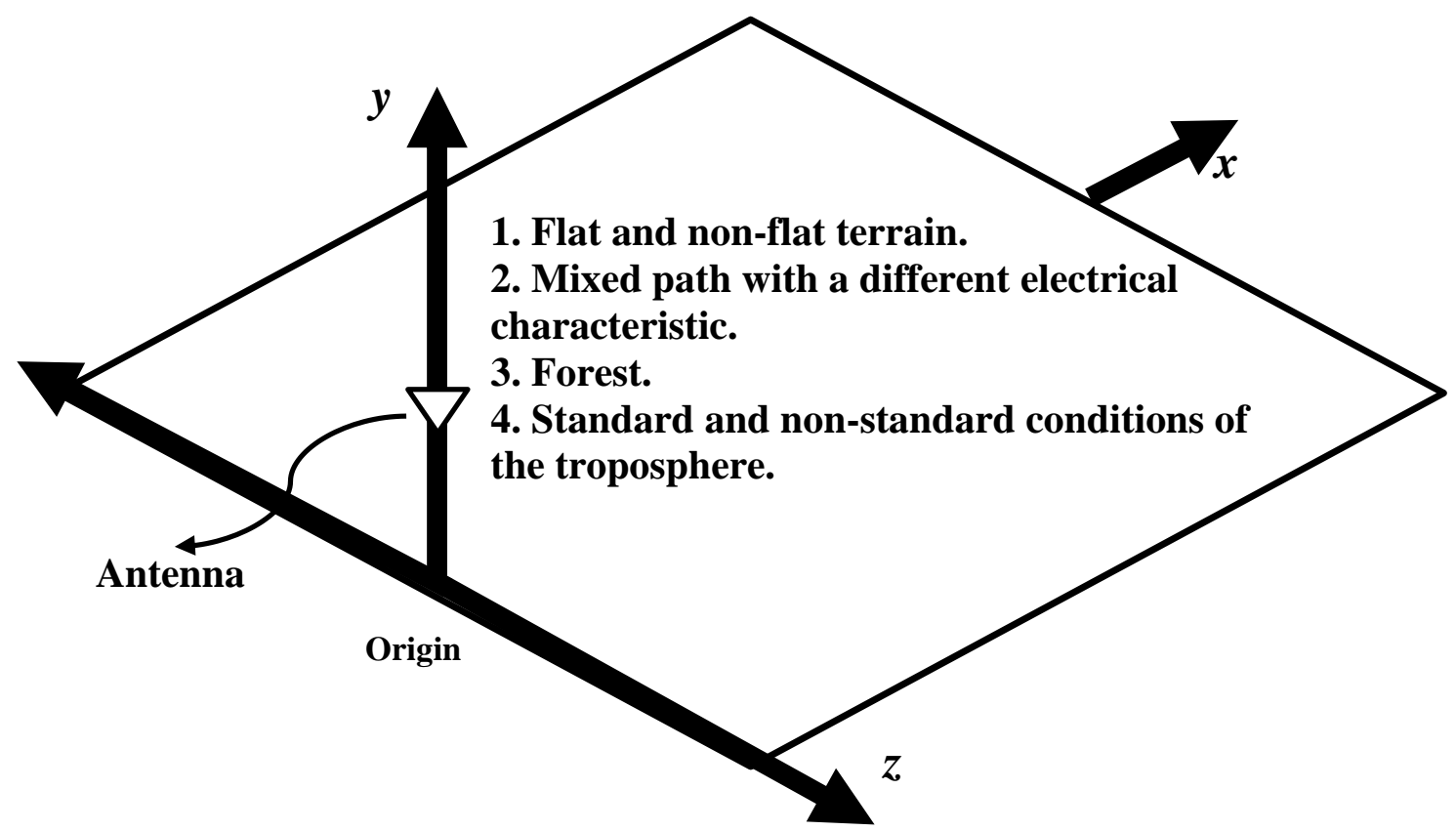

Figure 1. The 3D propagation area.

\subsection{Solution of 3D PE using higher order FDM}

Basically, solving (1) with BC (2) using higher order FDM requires the following steps:

Step 1: Dividing the solution region into a grid of nodes as shown in Figure 2. 

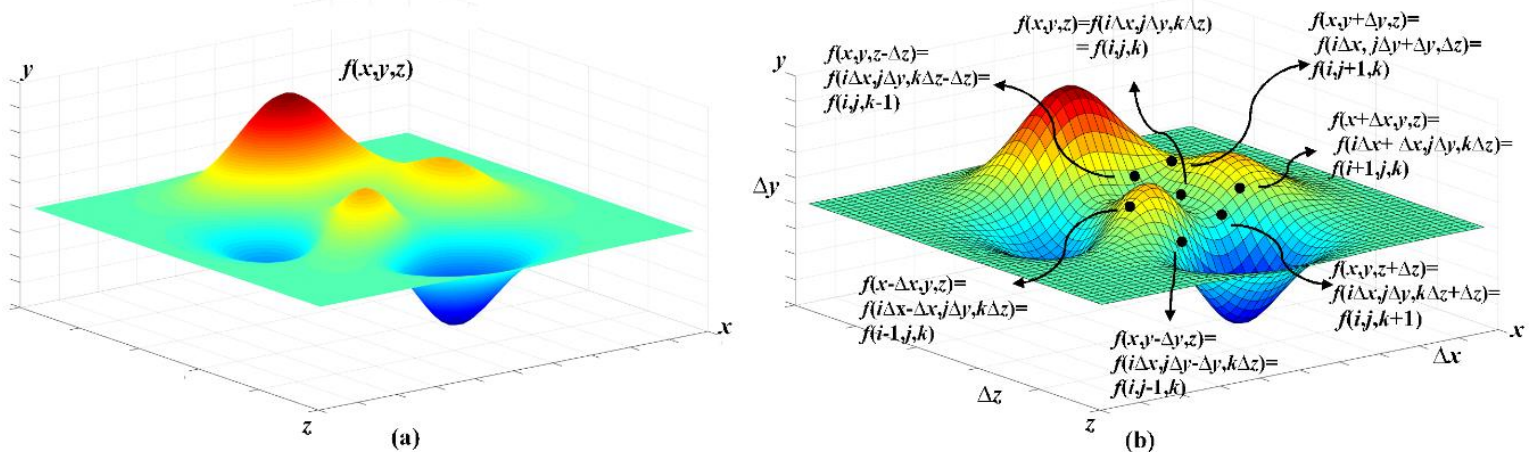

Figure 2. (a) Continuous function $f(x, y, z)$. (b) The discrete version of $f(x, y, z)$.

Step 2: Approximate the derivatives in (1) by finite difference [12] as follows:

The first derivative of $f(x, y, z)$ at $x$ is given by

$$
\begin{aligned}
& \left.\frac{\partial f(x, y, z)}{\partial x}\right|_{x} \approx \frac{f(x, y, z)-f(x-\Delta x, y, z)}{\Delta x}=\frac{f_{i, j, k}-f_{i-1, j, k}}{\Delta x} \\
& \left.\frac{\partial f(x, y, z)}{\partial x}\right|_{x} \approx \frac{3 f(x, y, z)-4 f(x-\Delta x, y, z)+f(x-2 \Delta x, y, z)}{2 \Delta x}=\frac{3 f_{i, j, k}-4 f_{i-1, j, k}+f_{i-2, j, k}}{2 \Delta x} \\
& \left.\frac{\partial f(x, y, z)}{\partial x}\right|_{x} \approx \frac{11 f(x, y, z)-18 f(x-\Delta x, y, z)+9 f(x-2 \Delta x, y, z)-2 f(x-3 \Delta x, y, z)}{6 \Delta x}=\frac{11 f_{i, j, k}-18 f_{i-1, j, k}+9 f_{i-2, j, k}-2 f_{i-3, j, k}}{6 \Delta x}
\end{aligned}
$$

The second derivative of $f(x, y, z)$ at $y$ is given by

$$
\begin{aligned}
\left.\frac{\partial^{2} f(x, y, z)}{\partial y^{2}}\right|_{y} & \approx \frac{f(x, y+\Delta y, z)-2 f(x, y, z)+f(x, y-\Delta y, z)}{(\Delta y)^{2}}=\frac{f_{i, j+1, k}-2 f_{i, j, k}+f_{i, j-1, k}}{(\Delta y)^{2}} \\
\left.\frac{\partial^{2} f(x, y, z)}{\partial y^{2}}\right|_{y} & \approx \frac{-f(x, y+2 \Delta y, z)+16 f(x, y+\Delta y, z)-30 f(x, y, z)+16 f(x, y-\Delta y, z)-f(x, y-2 \Delta y, z)}{12(\Delta y)^{2}} \\
& =\frac{-f_{i, j+2, k}+16 f_{i, j+1, k}-30 f_{i, j, k}+16 f_{i, j-1, k}-f_{i, j-2, k}}{12(\Delta y)^{2}}
\end{aligned}
$$

The second derivative of $f(x, y, z)$ at $z$ is given by

$$
\begin{aligned}
\left.\frac{\partial^{2} f(x, y, z)}{\partial z^{2}}\right|_{z} & \approx \frac{f(x, y, z+\Delta z)-2 f(x, y, z)+f(x, y, z-\Delta z)}{(\Delta z)^{2}}=\frac{f_{i, j, k+1}-2 f_{i, j, k}+f_{i, j, k-1}}{(\Delta z)^{2}} \\
\left.\frac{\partial^{2} f(x, y, z)}{\partial z^{2}}\right|_{z} & \approx \frac{-f(x, y, z+2 \Delta z)+16 f(x, y, z+\Delta z)-30 f(x, y, z)+16 f(x, y, z-\Delta z)-f(x, y, z-2 \Delta z)}{12(\Delta z)^{2}} \\
& =\frac{-f_{i, j, k+2}+16 f_{i, j, k+1}-30 f_{i, j, k}+16 f_{i, j, k-1}-f_{i, j, k-2}}{12(\Delta z)^{2}}
\end{aligned}
$$

Step 3: Combine (3.c), (4.b) and (5.b) with (1) as

$$
\begin{aligned}
& \frac{-f_{i, j, k+2}+16 f_{i, j, k+1}-30 f_{i, j, k}+16 f_{i, j, k-1}-f_{i, j, k-2}}{12(\Delta z)^{2}}+\frac{-f_{i, j+2, k}+16 f_{i, j+1, k}-30 f_{i, j, k}+16 f_{i, j-1, k}-f_{i, j-2, k}}{12(\Delta y)^{2}}+ \\
& 2 j k_{0} \frac{11 f_{i, j, k}-18 f_{i-1, j, k}+9 f_{i-2, j, k}-2 f_{i-3, j, k}}{6 \Delta x}+k_{0}^{2}\left(n^{2}-1\right) f_{i, j, k}=0 .
\end{aligned}
$$

Multiply above equation by $\frac{1}{11} \frac{6 \Delta x}{2 j k_{0}}$

$$
\begin{aligned}
& \frac{1}{11} \frac{6 \Delta x}{2 j k_{0}} \frac{-f_{i, j, k+2}+16 f_{i, j, k+1}-30 f_{i, j, k}+16 f_{i, j, k-1}-f_{i, j, k-2}}{12(\Delta z)^{2}}+\frac{1}{11} \frac{6 \Delta x}{2 j k_{0}} \frac{-f_{i, j+2, k}+16 f_{i, j+1, k}-30 f_{i, j, k}+16 f_{i, j-1, k}-f_{i, j-2, k}}{12(\Delta y)^{2}}+f_{i, j, k}- \\
& \frac{18}{11} f_{i-1, j, k}+\frac{9}{11} f_{i-2, j, k}-\frac{2}{11} f_{i-3, j, k}+\frac{1}{11} \frac{6 \Delta x}{2 j k_{0}} k_{0}^{2}\left(n^{2}-1\right) f_{i, j, k}=0 .
\end{aligned}
$$

Put the previous values of $f$ in one side and the future value of $f$ in the other side of the equation 
$\frac{18}{11} f_{i-1, j, k}-\frac{9}{11} f_{i-2, j, k}+\frac{2}{11} f_{i-3, j, k}=\frac{1}{11} \frac{6 \Delta x}{2 j k_{0}} \frac{-f_{i, j, k+2}+16 f_{i, j, k+1}-30 f_{i, j, k}+16 f_{i, j, k-1}-f_{i, j, k-2}}{12(\Delta z)^{2}}+$
$\frac{1}{11} \frac{6 \Delta x}{2 j k_{0}} \frac{-f_{i, j+2, k}+16 f_{i, j+1, k}-30 f_{i, j, k}+16 f_{i, j-1, k}-f_{i, j-2, k}}{12(\Delta y)^{2}}+\left(\frac{1}{11} \frac{6 \Delta x}{2 j k_{0}} k_{0}^{2}\left(n^{2}-1\right)+1\right) f_{i, j, k}$

From (6), it is clear the present value of $f$ depends on the three previous values of $f$.

Step 4: Write (6) in matrix form as

$\frac{1}{11} \frac{6 \Delta x}{2 j k_{0}}\left[D_{z}^{2}\right][f]+\frac{1}{11} \frac{6 \Delta x}{2 j k_{0}}\left[D_{y}^{2}\right][f]+\left(\frac{1}{11} \frac{6 \Delta x}{2 j k_{0}} k_{0}^{2}\left(n^{2}-1\right)+1\right)[I][f]=[g]$

where $[f]=\left[\begin{array}{c}f_{i, 1,1} \\ \vdots \\ f_{i, M, N}\end{array}\right],[g]=\frac{18}{11}\left[\begin{array}{c}f_{i-1,1,1} \\ \vdots \\ f_{i-1, M, N}\end{array}\right]-\frac{9}{11}\left[\begin{array}{c}f_{i-2,1,1} \\ \vdots \\ f_{i-2, M, N}\end{array}\right]+\frac{2}{11}\left[\begin{array}{c}f_{i-3,1,1} \\ \vdots \\ f_{i-3, M, N}\end{array}\right], \quad[I]$ is the identity matrix, $M$ and $N$ are the last point on the grid in the $y$ and $z$ directions, $\left[D_{z}^{2}\right]$ is a sparse matrix with values $\frac{-30}{12 \Delta z^{2}}$ on the main diagonal, $\frac{16}{12 \Delta z^{2}}$ on first diagonal above and below the main diagonal, $\frac{-1}{12 \Delta z^{2}}$ on second diagonal above and below the main diagonal, and $\left[D_{y}^{2}\right]$ is a sparse matrix with values $\frac{-30}{12 \Delta y^{2}}$ on the main diagonal, $\frac{16}{12 \Delta y^{2}}$ on the $M^{\text {th }}$ diagonal above and below the main diagonal, and $\frac{-1}{12 \Delta y^{2}}$ on the $2 M^{\text {th }}$ diagonal above and below the main diagonal. Note that the elements in $\left[D_{y}^{2}\right]$ and $\left[D_{z}^{2}\right]$ are filled using (4.b) and (5.b) respectively. Also, zero is put in place of any point outside the grid.

Step 5: Factor out $[f]$ to put (7) in standard form $\mathbf{A f}=\mathbf{g}$. Equation (7) becomes

$$
\left(\frac{1}{11} \frac{6 \Delta x}{2 j k_{0}}\left[D_{z}^{2}\right]+\frac{1}{11} \frac{6 \Delta x}{2 j k_{0}}\left[D_{y}^{2}\right]+\left(\frac{1}{11} \frac{6 \Delta x}{2 j k_{0}} k_{0}^{2}\left(n^{2}-1\right)+1\right)[I]\right)[f]=[g]
$$

Where,

$\mathbf{A}=\left(\frac{1}{11} \frac{6 \Delta x}{2 j k_{0}}\left[D_{z}^{2}\right]+\frac{1}{11} \frac{6 \Delta x}{2 j k_{0}}\left[D_{y}^{2}\right]+\left(\frac{1}{11} \frac{6 \Delta x}{2 j k_{0}} k_{0}^{2}\left(n^{2}-1\right)+1\right)[I]\right)$

Step 6: Incorporate BCs, i.e. (2), into A using the following steps:

a. For each row in $\mathbf{A}$ that corresponds to a boundary point, replace entire row with 0's as

i. For surface $\mathrm{BC}$, the rows numbers are $n=(M \times N-M+1): 1: M \times N$.

b. Apply the BCs as

i. For HP BC:

1. Insert a 1 into the $[A]_{n, n}$ where $n$ is equal to the same rows numbers in (a).

2. Place the boundary value $C$ in the same rows numbers of $\mathbf{g}$.

ii. For mixed BC:

1. It is at the surface. Equation (2) is in FDM $\cong \frac{f_{i, 1, k}-f_{i, 2, k}}{\Delta y}+\gamma$. Therefore, insert $\frac{1}{\Delta y}+\gamma$ into the $[A]_{n . n}$ and $-\frac{1}{\Delta y}$ into the $[A]_{n, n+M}$ where $n$ is equal to the same rows numbers in (a.(i)).

2. Place the boundary value $C$ in the same rows numbers of $\mathbf{g}$.

iii. $\mathrm{VP} \mathrm{BC}$ is mixed $\mathrm{BC}$ with $\gamma=0$.

Step 7: Solve the matrix equation $[A][f]=[g]$.

Note that to be able to use (7), the values of $f_{1, j, k}, f_{2, j, k}$, and $f_{3, j, k}$ have to be known. $f_{1, j, k}$ is the initial values of the field which obtained via antenna's pattern. To find $f_{2, j, k}$, we re-derive (7) by replacing (3.c) by (3.a). The solution for the new equation will be $f_{2, j, k}$. In the same manner $f_{3, j, k}$ is found. We re-derive (7) by replacing (3.c) by (3.b). The solution for the new equation will be $f_{3, j, k}$. After that, (7) can be used to solve the problem.

\subsection{Upper and sides boundaries}

Since the propagation problem unbounded when $\mathrm{y} \rightarrow \infty, \mathrm{z} \rightarrow-\infty$ and $\mathrm{z} \rightarrow \infty$, an absorption $\mathrm{BC}$ is required at specific height and width. Perfectly Matched Layer method is used in this paper for that purpose [13]. 


\subsection{Initial field}

The initial field in the spatial domain can be represented as $f(0, y, z)=A\left(0, y-y_{s}, z-z_{s}\right)+$ $\Gamma A^{*}\left(0, y+y_{s}, z+z_{s}\right)$, where $A$ is antenna aperture distribution, $A^{*}$ is the image antenna aperture distribution, $y_{s}$ and $z_{s}$ are the location of the antenna from the origin in $y$ and $z$ directions respectively, and $\Gamma$ is the reflection coefficient. For PEC, $\Gamma=-1$, and 1 for HP and VP respectively. For lossy ground, $\Gamma=\frac{\sin \theta-\sqrt{\varepsilon-\cos ^{2} \theta}}{\sin \theta+\sqrt{\varepsilon-\cos ^{2} \theta}}$ for HP and $\Gamma=\frac{\varepsilon \sin \theta-\sqrt{\varepsilon-\cos ^{2} \theta}}{\varepsilon \sin \theta+\sqrt{\varepsilon-\cos ^{2} \theta}}$ for VP where $\theta$ is the angle of incidence.

\subsection{Different types of terrains and environments}

\subsubsection{Non-flat terrain}

The approach used here is presented in [2]. First, a sequence of down and up stair steps approximates the irregular terrain. Then, on each step, the perpendicular field is evaluated in the normal way, forcing the chosen BCs on the ground surface. Finally, zeros are placed inside the field values of the terrain.

\subsubsection{Mixed path}

In some real environments, the wave propagates over several types of ground sections in such a way that each section has different electrical characteristics. To deal with such environment, update and fix electrical characteristic within the boundary, i.e. (2), of each section in the solution.

\subsubsection{Forest environment}

Two approaches are used to include forest environment to the PE. The first approach approximates the forest by one dielectric slab as shown in Figure 3.a [14]. This approach is useable for frequency range from 20 to $200 \mathrm{MHz}$. The other approach approximates the forest by three dielectric slabs; ground, trunk, and canopy slab as shown in Figure 3.b. [15]. This model is valid for frequency band from $200 \mathrm{MHz}$ to $2 \mathrm{GHz}$. The dielectric slab for both models is given by complex refractive index $n=\sqrt{\varepsilon_{r}-j \sigma / \omega \varepsilon_{0}}$ where $\varepsilon_{r}$ and $\sigma$ are relative permittivity and conductivity of forest dielectric slab layer respectively.

\begin{tabular}{llll} 
Layer 1 Troposphere & $\frac{\text { Layer } 1 \quad \text { Troposphere }}{\text { Layer } 2} \operatorname{Canopy}\left(\varepsilon_{r 2}, \sigma_{2}\right)$ \\
\hline Layer $2 \quad$ Canopy $\left(\varepsilon_{r 2}, \sigma_{2}\right)$ & Layer $3 \quad \operatorname{Trunk}\left(\varepsilon_{r 3}, \sigma_{3}\right)$ \\
\hline
\end{tabular}

(a)

(b)

Figure 3. (a) Model of 1-layer. (b) Model of 3-layers.

\section{RESULTS AND DISCUSSION}

To validate the proposed method, the mean square error (MSE) is calculated for the proposed approach which is define as

$$
\mathrm{MSE}=\frac{\sum_{j=1}^{N}\left[R_{j}^{F D M}-R_{j}^{r e f}\right]^{2}}{\sum_{j=1}^{N}\left[R_{j}^{r e f}\right]^{2}}
$$

where $R_{j}^{F D M}$ and $R_{j}^{r e f}$ are the results that have been gotten from higher order FDM and a reference solution of the parabolic equation respectively. The two-dimensional (2D) Fourier split-step (FSS) solution method is used as a reference solution.

\subsection{Propagation over perfectly flat surfaces under standard and non-standard atmosphere}

The propagation of a HP electromagnetic (EM) wave radiated by an omni-directional antenna over PEC flat surface under standard atmosphere $(M(x, y, z)=0.118 y)$ and non-standard atmosphere $(\partial M(x, y, z)$ $/ \partial y=0.3$ for $0<y<50, \partial M(x, y, z) / \partial y=-0.15$ for $50<y<150$, and $\partial M(x, y, z) / \partial y=0.118$ for $y>150)$ are shown in Figure 4 and Figure 5, respectively. The antenna is located at height of $100 \mathrm{~m}$ in the $y$-direction and $0 \mathrm{Km}$ in the $z$ direction, and it operates at $1 \mathrm{GHz}$. The maximum range and width are $200 \mathrm{Km}$. The coverage diagram of field 
at the middle (cross section $0<x<200 \mathrm{Km}, 0<y<300 \mathrm{~m}$, and $z=0 \mathrm{Km}$ ) is shown in Figure 4.a and Figure 5.a respectively. The field as a function of height at ranges 50,100,150, and $200 \mathrm{Km}$ from the transmitter is shown in Figure 4.b and Figure 5.b respectively. Also, the field as a function of range at heights of 100, 200, 300, and $400 \mathrm{~m}$ from the surface is shown in Figure 4.c and Figure 5.c respectively. The results are compared with 2D FSS. Excellent agreement appears between the results and MSE $=9.46 \times 10^{-6}$ for Figure 4 and MSE $=$ $8.43 \times 10^{-6}$ for Figure 5 .
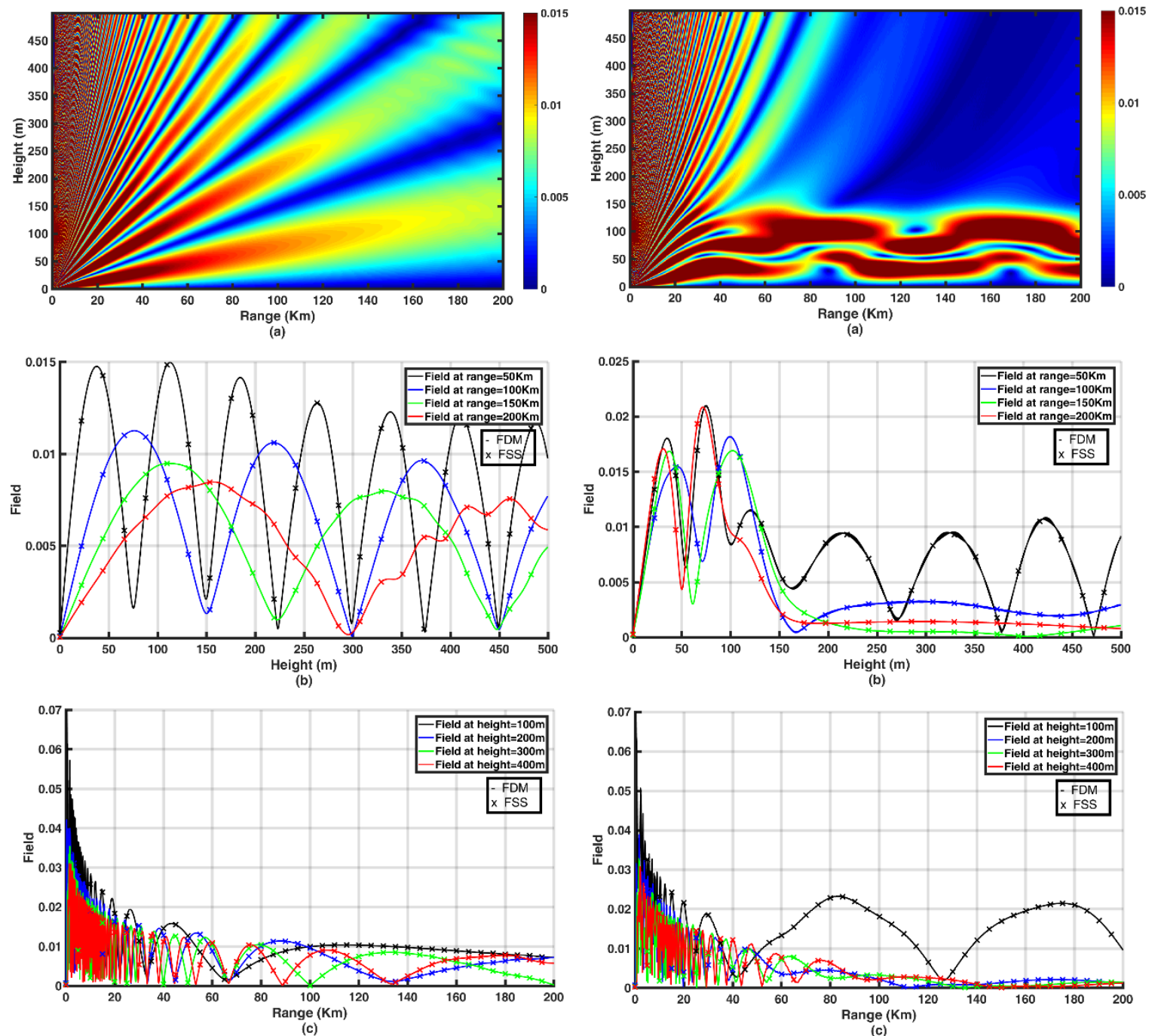

Figure 4. (a) Coverage diagram of the field. (b) The field as a function of height. (c) The field as a function of range.

Figure 5. (a) Coverage diagram of the field. (b) The field as a function of height. (c) The field as a function of range.

\subsection{Propagation over finitely mixed flat surfaces}

The propagation of a HP EM wave radiated by Gaussian antenna over mixed path; sea-land-sea, with electrical characteristics, $\varepsilon_{r \text { sea }}=80, \sigma_{\text {sea }}=5$ siemens $/ \mathrm{m}, \varepsilon_{r \text { land }}=10$, and $\sigma_{\text {land }}=0.002$ siemens $/ \mathrm{m}$, is shown Figure 6. Each segment has a length of $5 \mathrm{Km}$. The antenna is located at a height of $100 \mathrm{~m}$ in the $y$ direction and $0 \mathrm{Km}$ in the $z$-direction, and it operates at $30 \mathrm{MHz}$ with beamwidth and elevation angles in both $y$ and $z$ directions are equal to $1^{\circ}$ and $0^{\circ}$ respectively, with refractive index of 1 (free space). The coverage diagram of field at the middle (cross section $0<x<15 \mathrm{Km}, 0<y<500 \mathrm{~m}$, and $z=0 \mathrm{Km}$ ) is shown in Figure 6.a. The field as a function of height at ranges $2.5,7.5$, and $12.5 \mathrm{Km}$ from the transmitter is shown in Figure $6 . \mathrm{b}$ and compared with 2D FSS. Also, the field as a function of range at heights of 100, 200, 300, and 400m from the surface is shown in Figure 6.c and compared with 2D FSS. The MSE $=2.95 \times 10^{-6}$. 


\subsection{Propagation over non-flat terrain}

The propagation of a HP EM wave radiated by Gaussian antenna over PEC non-flat terrain surface is shown Figure 7. The antenna is located at a height of $100 \mathrm{~m}$ in the $y$-direction and $0 \mathrm{Km}$ in the $z$-direction, and it operates at $300 \mathrm{MHz}$ with beamwidth and elevation angles in $y$ and $z$ directions are $1^{\circ}$ and $0^{\circ}$ respectively, with refractive index of $n=1$ (free space). The maximum range and width are $50 \mathrm{Km}$. The coverage diagram of field at the middle (cross section $0<x<50 \mathrm{KM}, 0<y<500 \mathrm{~m}$, and $z=0 \mathrm{Km}$ ) is shown in Figure 7.a. The field as a function of height at ranges 10, 20,30,40, and 50Km from the transmitter is shown in Figure 7.b and compared with 2D FSS. Also, the field as a function of range at heights of 100, 200, 300, and 400m from the surface is shown in Figure 7.c and compared with 2D FSS. The MSE $=6.37 \times 10^{-6}$.

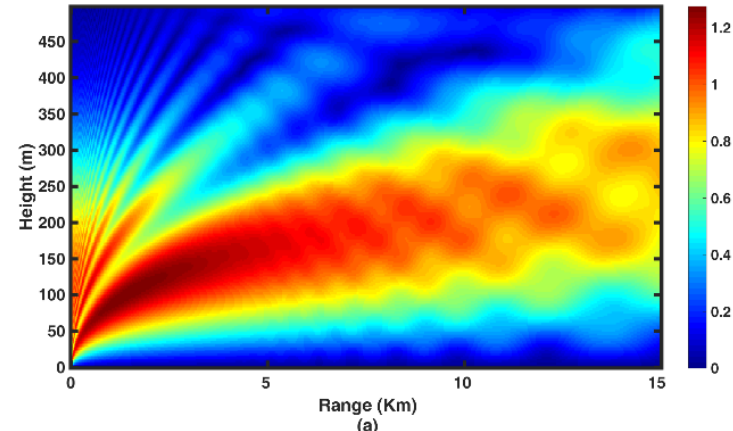

(a)
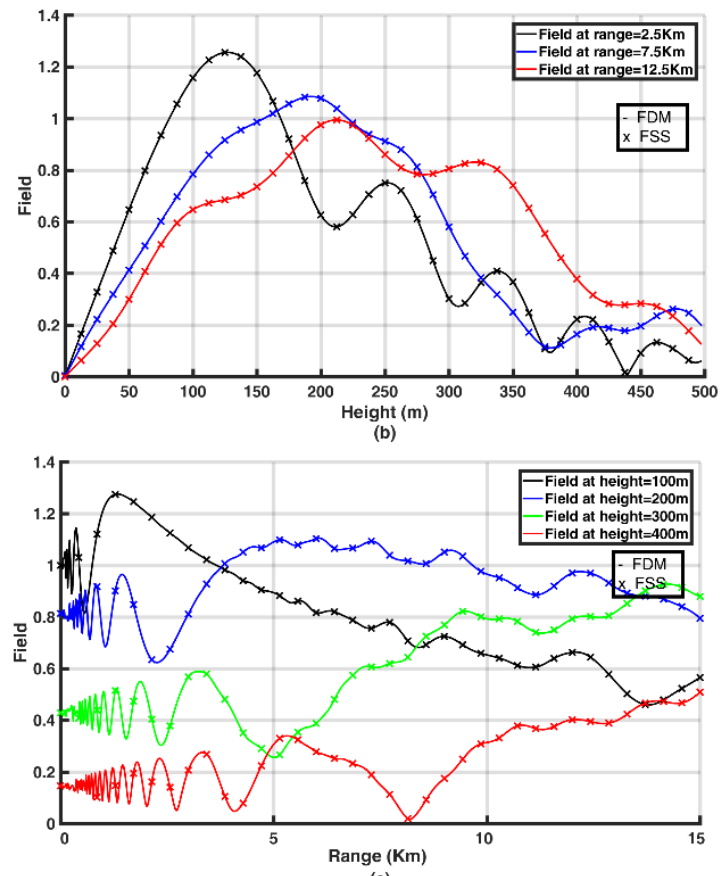

Figure 6. (a) Coverage diagram of the field. (b) The field as a function of height. (c) The field as a function of range.
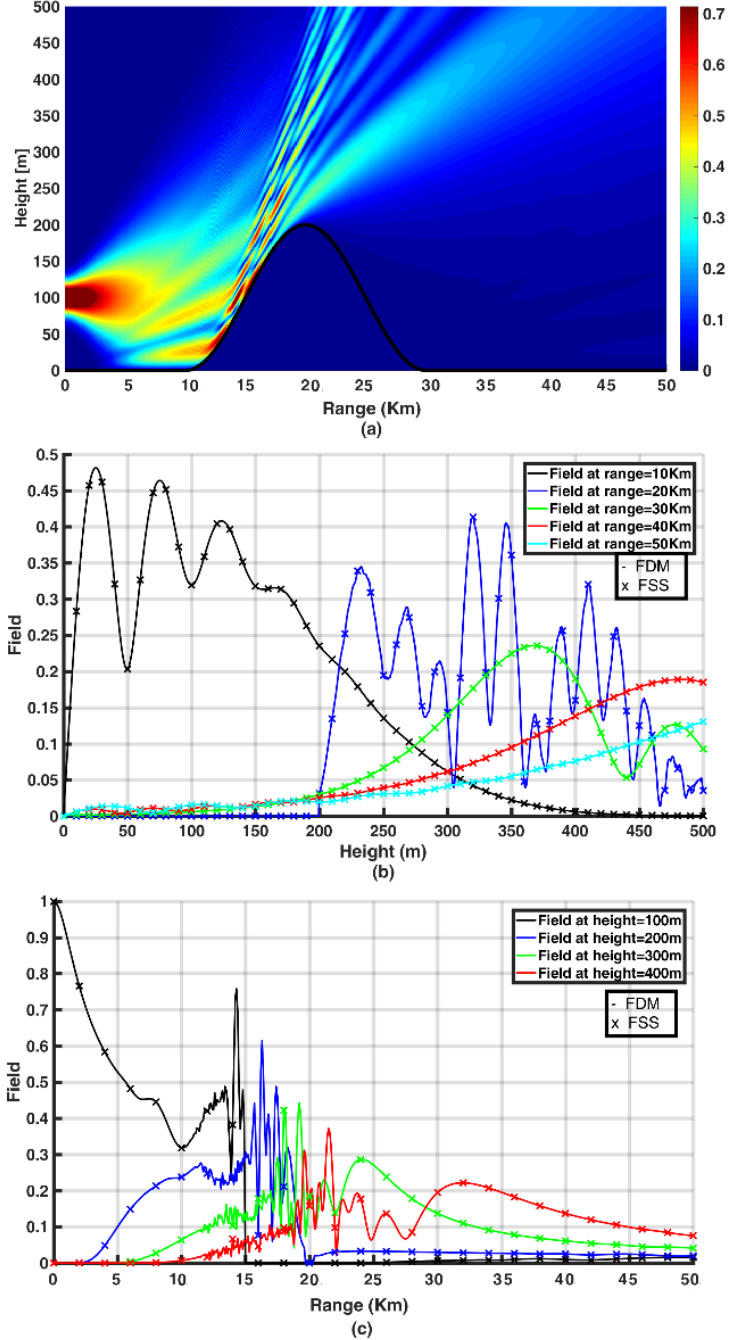

Figure 7. (a) Coverage Diagram of the field. (b) The field as a function of height. (c) The field as a function of range.

\subsection{Forest environment}

The propagation loss (PL) (defined as the ratio between the power radiated by the transmitting antenna and the power which would be available at the receiving antenna) of HP EM wave radiated by omni-directional antenna operating at $50 \mathrm{MHz}$ located at a height of $5 \mathrm{~m}$ and $0 \mathrm{Km}$ in the $y$ and $z$ directions respectively, with $M(x, y, z)=0.118 y$ outside the forest is shown in Figure 8. The maximum range is $40 \mathrm{Km}$ and the forest extends from 0 to $40 \mathrm{Km}$ in $x$ and $z$ directions. The altitude of the trees is $18 \mathrm{~m}$. The relative permittivity for the forest is $\varepsilon_{r}=1.5$ and the conductivity is $\sigma=1 \times 10^{-3}$ siemens $/ \mathrm{m}$. The coverage diagram of the PL at the middle (cross section $0<x<40 \mathrm{Km}, 0<y<100 \mathrm{~m}$, and $z=0 \mathrm{Km}$ ) is shown in Figure 8.a. The PL as a function of height at ranges 10, 20,30, and 40Km from the transmitter is shown in Figure 8.b and compared with 2D FSS. Also, the 
PL as a function of range at heights of 20,40,60, and 80m from the surface is shown in Figure 8.c and compared with 2D FSS. The MSE $=4.03 \times 10^{-6}$.

Next, the field of HP EM wave radiated by omni-directional antenna operating at $1 \mathrm{GHz}$ located at a height of $30 \mathrm{~m}$ and $0 \mathrm{Km}$ in the $y$ and $z$ direction respectively, with $M(x, y, z)=0.118 y$ outside the forest is shown in Figure 9. The maximum range and width are $5 \mathrm{Km}$. The forest extends form $0 \mathrm{Km}$ to $5 \mathrm{Km}$ in $x$ and $z$ directions respectively. The altitude of the trees is $25 \mathrm{~m}$. The electrical characteristics for the forest are assumed to be $\varepsilon_{r}=20, \sigma=10 \times 10^{-3}$ siemens $/ \mathrm{m}$ for the ground, $\varepsilon_{r}=1.03, \sigma=0.03 \times 10^{-3}$ siemens $/ \mathrm{m}$ for the trunk with $15 \mathrm{~m}$ height, and $\varepsilon_{r}=1.12, \sigma=0.12 \times 10^{-3}$ siemens $/ \mathrm{m}$ for the canopy with $10 \mathrm{~m}$ height. The coverage diagram of PL at the middle (cross section $0<x<5 \mathrm{Km}, 0<y<100 \mathrm{~m}$, and $z=0 \mathrm{Km}$ ) is shown in Figure 9.a. The PL as a function of height at ranges $1,2,3$, and $4 \mathrm{Km}$ from the transmitter is shown in Figure 9.b and compared with 2D FSS. Also, the PL as a function of range at heights of 20,40,60, and 80m from the surface is shown in Figure 9.c and compared with 2D FSS. The MSE $=1.22 \times 10^{-6}$.
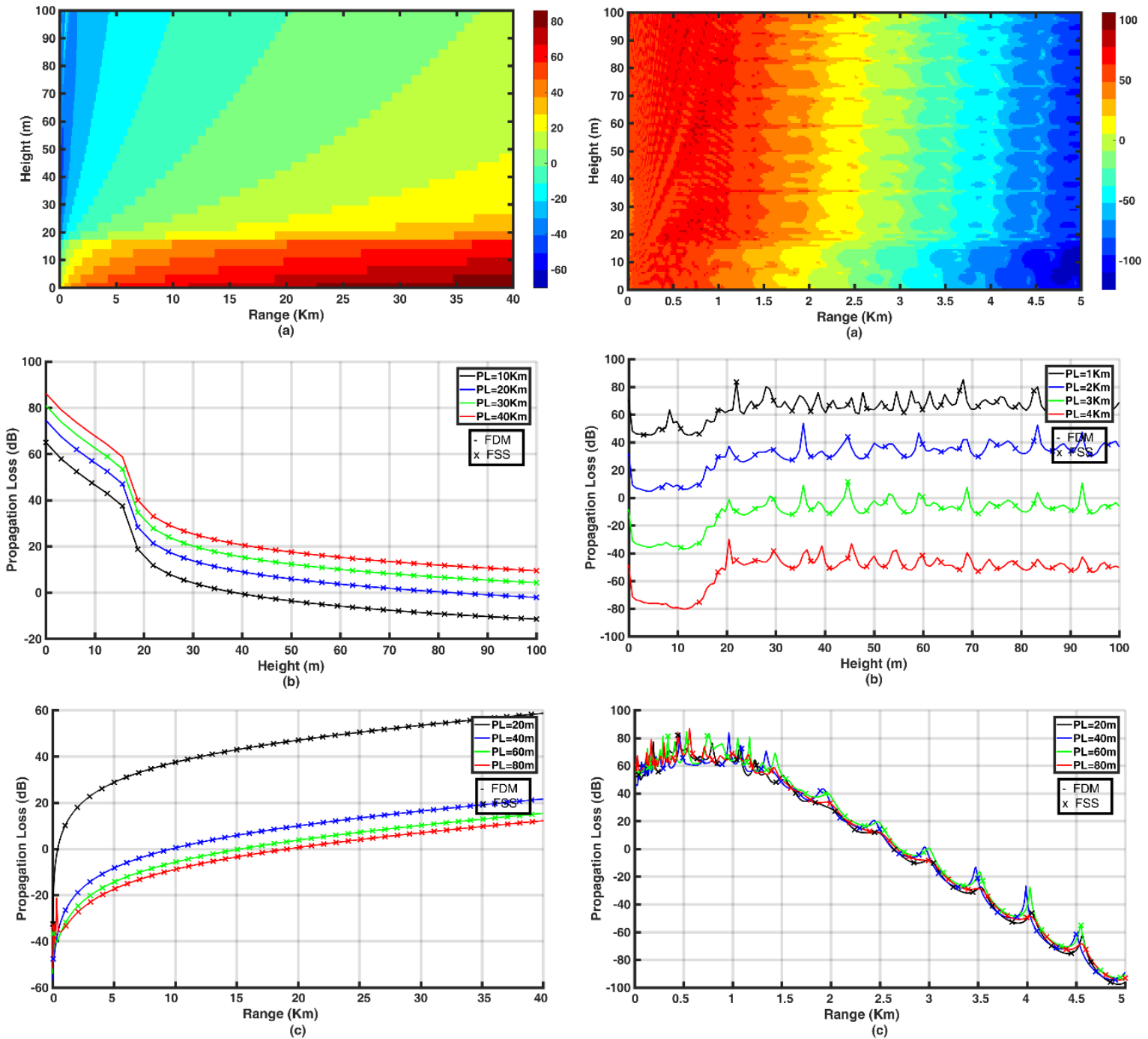

Figure 8. (a) Coverage diagram of the PL. (b) The PL as a function of height. (c) The PL as a function of range.

Figure 9. (a) Coverage diagram of the PL. (b) The PL as a function of height. (c) The PL as a function of range.

\section{CONCLUSION}

In this paper, modeling of the HP and VP EM waves radiated by an antenna with specific characteristics; frequency, radiation pattern, altitude, beamwidth and elevation angles, through standard and non-standard refractive index of troposphere; over perfectly and finitely conductive mixed surfaces, flat and non-flat surfaces and forest environment was performed using higher order FDM solution of the 3D PE. 


\section{REFERENCES}

[1] M. Levy, Parabolic Equation Methods for Electromagnetic Wave Propagation. The institution of Electrical Engineerings, Michael Faraday House, London United Kingdom, 2000.

[2] Gökhan Apaydin and Levent Sevgi, Radio wave propagation and parabolic equation modeling, New Jersey, John Wiley \& Sons, Inc., 2017.

[3] C. A. Zelley and C. C. Constantinou, "A three-dimensional parabolic equation applied to VHF/UHF propagation over irregular terrain," IEEE Transcations on Antennas Propagation, vol. 47, no. 10, pp. 1586-1596, 1999.

[4] A. A. Zaporozhets, "Application of vector parabolic equation method to urban radiowave propagation problems," IEE Proc.-Microwave., Antennas Propagation, vol. 146, no. 4, pp. 253-256, 1999.

[5] R. Janaswamy and J. B. Andersen, "Path loss predictions in urban areas with irregular terrain topography," Wireless Pers. Commutation., vol. 12, no. 3, pp. 255-268, 2000.

[6] R. Janaswamy, "Path loss predictions in the presence of buildings on flat terrain: A 3-D vector parabolic equation approach," IEEE Transcations on Antennas Propagation, vol. 51, no. 8, pp. 1716-1728, Aug. 2003.

[7] M. A. N. Silva, E. Costa, and M. Liniger, "Analysis of the effects of irregular terrain on radio wave propagation based on a three-dimensional parabolic equation," IEEE Transcations on Antennas Propagation, vol. 60, no. 4, pp. 2138-2143, Apr. 2012.

[8] R. S. Awadallah, J. Z. Gehman, J. R. Kuttler, and M. H. Newkirk, "Effects of lateral terrain variations on tropospheric radar propagation," IEEE Transcations on Antennas Propagation, vol. 53, no. 1, pp. 420-434, 2005.

[9] Zeina El Ahdab, and Funda Akleman, "Radiowave Propagation Analysis With a Bidirectional 3-D Vector Parabolic Equation Method," IEEE Transcations on Antennas Propagation, vol. 65, no. 4, pp. 1958-1966, Jan. 2017.

[10] Othmane Benhmammouch, Natacha Caouren, Ali Khenchaf, "Modeling of Roughness Effects on Electromagnetic Waves Propagation above Sea Surface Using 3D Parabolic Equation," IEEE International Geoscience and Remote Sensing Symposium, Cape Town, South Africa, Vol. 2, pp. II-817-II-820, 12-17 July 2009.

[11] V. A. Permyakov, M. S. Mikhailov, and E. S. Malevich, "Analysis of propagation of electromagnetic waves in difficult conditions by the parabolic equation method," IEEE Trans. Antennas Propagation, vol. 67, no. 4, pp. 2167-2175, Apr. 2019.

[12] David Eberly, Derivative Approximation by Finite Differences, Geometric Tools, Redmond WA, 2020.

[13] J. P. Berenger, "A perfectly matched layer for the absorption of electromagnetic waves," Journal Computational Physcs, vol. 114, no. 2, pp. 185-200, 1994.

[14] T. Tamir, "On Radio-Wave Propagation in Forest Environments," IEEE Transactions on Antennas and Propagation, vol. 15, np. 6, 806-817, 1967.

[15] Seker, S. S. "VHF/UHF Radiowave Propagation through Forests: Modeling and Experimental Observations," IEE Proceedings H - Microwaves, Antennas and Propagation, vol.139, no. 1, 72-78, 1992. 